\title{
Pigmentary and photonic coloration mechanisms reveal taxonomic relationships of the Cattlehearts (Lepidoptera: Papilionidae: Parides)
}

\author{
Bodo D Wilts ${ }^{1,2^{*}}$, Natasja IJbema ${ }^{1,3}$ and Doekele G Stavenga ${ }^{1}$
}

\begin{abstract}
Background: The colorful wing patterns of butterflies, a prime example of biodiversity, can change dramatically within closely related species. Wing pattern diversity is specifically present among papilionid butterflies. Whether a correlation between color and the evolution of these butterflies exists so far remained unsolved.

Results: We here investigate the Cattlehearts, Parides, a small Neotropical genus of papilionid butterflies with 36 members, the wings of which are marked by distinctly colored patches. By applying various physical techniques, we investigate the coloration toolkit of the wing scales. The wing scales contain two different, wavelength-selective absorbing pigments, causing pigmentary colorations. Scale ridges with multilayered lamellae, lumen multilayers or gyroid photonic crystals in the scale lumen create structural colors that are variously combined with these pigmentary colors.

Conclusions: The pigmentary and structural traits strongly correlate with the taxonomical distribution of Parides species. The experimental findings add crucial insight into the evolution of butterfly wing scales and show the importance of morphological parameter mapping for butterfly phylogenetics.
\end{abstract}

Keywords: Iridescence, Morphology, Papilionidae, Papiliochrome, Phylogeny, Scattering, Wing traits

\section{Background}

The wing coloration and patterning of a butterfly play important roles in its everyday survival. The coloration pattern can function as a concealment or disguise signal for protection (camouflage, crypsis) or for attracting potential mates (display) [1,2]. Coloration furthermore is an important aspect in mimicry, i.e. the parallel evolution of species that have evolved optically similar traits. Indeed, color is an important element of species selection mechanisms as many species have evolved by means of diverging coloration [3]. Evolutionary mechanisms of coloration appeared independently in multiple phylogenetic places, contributing largely to the rich biodiversity of life present on earth today [4]. Butterflies are a prime example of biodiversity [5,6]. Even though extensively

\footnotetext{
* Correspondence: bdw36@cam.ac.uk

${ }^{1}$ Computational Physics, Zernike Institute for Advanced Materials, University of Groningen, Nijenborgh 4, NL-9747AG Groningen, The Netherlands ${ }^{2}$ Present address: Department of Physics, Cavendish Laboratories, University of Cambridge, $13 \mathrm{JJ}$ Thomson Avenue, CB3 OHE Cambridge, UK Full list of author information is available at the end of the article
}

investigated in the past, it remains unclear whether their phylogeny and wing color are correlated.

Here, we investigate a possible color-phylogeny correlation in a small genus of papilionid butterflies, the Cattlehearts (Parides; Huebner, 1819), a Neotropical New World taxon with 36 members $[7,8]$. The coloration of Parides butterflies is quite stunning and diverse (Figure 1). The wings are predominantly black, but distinct wing patches feature vividly colored scales that can strongly differ between species. The colors and arrangement of these brightly colored spots on the butterfly wings serve as an aposematic signal to birds [9]. Aposematism is an antipredatory strategy where a potential prey radiates its unpalatability by a signal that is readily understood by predators [10]. With their prominent color patterns, Parides butterflies are suitable for Müllerian mimicry rings. Their predators - especially birds - quickly learn to recognize these patterns and become averse to it [11].

Coloration is generally divided into two classes: pigmentary (chemical) coloration, due to wavelength-selective light absorption by pigments, and structural (physical) 


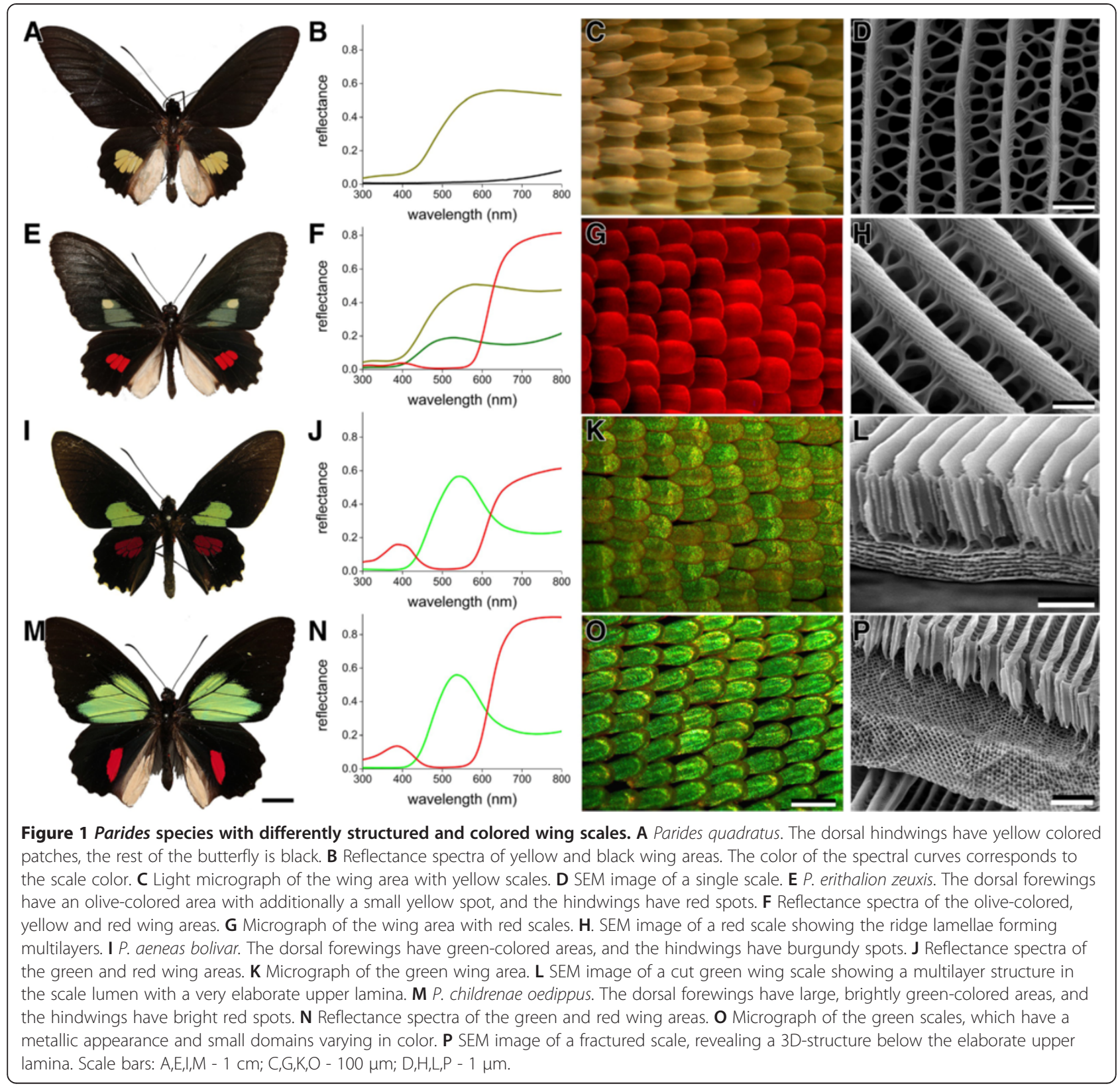

coloration, due to wavelength-selective light reflection resulting from incident light interacting with nanostructured matter [12]. Up to date, of the Cattlehearts only the coloration principles of the Emerald-Patched Cattleheart, $P$. sesostris, have been elucidated in extensive detail [13-20]. The wing scales of the green patches at the dorsal wings of $P$. sesostris contain a single-network, gyroid-type photonic crystal layer, which reflects blue-green light, depending on the angle of illumination. However, the scales also contain a violet-absorbing pigment that functions as a spectral filter, causing an angle-independent, vivid-green wing color [19].

The small size of the genus with its large diversity in wing coloration makes the Parides an attractive study group for investigating possible color-phylogeny relationships. By applying various physical techniques [21], we investigated the coloration mechanisms of the male members of the genus Parides. We thus identified a restricted set of pigments and photonic structures that together create the vivid colors of Parides butterflies. We discuss the results in view of the evolution of butterfly wing scales and the taxonomic relationships of coloration in phylogenetic trees.

\section{Results}

Appearance of the wings and wing scale morphology The dorsal wings of Parides butterflies feature brightly colored patches in a generally jet-black frame [22,23]. 
Figure 1 presents an overview of the wing patterns of a few typical Parides species (Figure 1A,E,I,M). To investigate the nature of the variously colored wing areas, we measured reflectance spectra with a bifurcated probe (Figure 1B,F,J,N), and we made light micrographs of the scales in the colored wing areas (Figure 1C,G,K,O). We further performed scanning electron microscopy (SEM) to reveal the ultrastructure of the scales (Figure $1 \mathrm{D}, \mathrm{H}$, L,P).

The dorsal wings of $P$. quadratus (Figure 1A) are largely black with small yellow patches in the hindwings. As expected for its yellow color, the reflectance spectrum of the wing patches is low in the ultraviolet and high in the long-wavelength range (Figure 1B). The reflectance of the black wing areas is minimal in the ultraviolet and visible wavelength ranges but becomes noticeable in the far-red, which is characteristic for high concentrations of melanin (Figure 1B). The yellow scales are densely stacked in a regular lattice (Figure 1C). Scanning electron microscopy shows that the scales are built according to the basic bauplan of papilionid butterfly wing scales [23,24]; the upper surface features parallel ridges, with in between randomly arranged cross-ribs, which form large windows (Figure 1D).

The dorsal wings of $P$. erithalion zeuxis (Figure 1E) display various colors: olive, yellow, and red. The reflectance spectra vary accordingly (Figure 1F) and indicate different pigments as well as scale structures. The wing patches of the hindwings are covered by densely stacked red scales (Figure 1G) The anatomy of the wing scales is similar to that shown in Figure 1D, but the ridges are taller due to a large stack of overlapping ridge lamellae, which together create a slender, highly-tilted multilayer (Figure $1 \mathrm{H}$ ). The multilayered scales show, with normal illumination of the wing, a strong blue-iridescence in an oblique direction (see below, Figure 2).

The dorsal forewings of $P$. aeneas bolivar (Figure 1I) have green patches and the hindwings red patches. Their reflectance spectra contrast strongly (Figure 1J). The quite pronounced green reflectance indicates a structural origin, which is also suggested by the gleam of the wing scales (Figure $1 \mathrm{~K}$ ). Indeed, scanning electron microscopy revealed elaborately structured scales (Figure 1L). The scale lumen consists of a large multilayer, and the scale's upper lamina has tall ridges connected with a thick layer of cross-ribs, quite in contrast with the basic pattern of moderate ridges and thin cross-ribs (Figure 1D).

Of the species shown in Figure 1, P. childrenae oedippus (Figure 1M) has the most striking color pattern. The reflectance spectra of the green and red patches are similar to those of $P$. aeneas, suggesting similar coloration mechanisms. However, with epi-illumination light microscopy the green scales have a rather different optical appearance, especially a different gloss, indicating the presence of dissimilar photonic structures (Figure 1O). Scanning electron microscopy of a fractured scale revealed a 3D network-like structure in the scale lumen (Figure 1P), which was previously demonstrated to be a gyroid-type photonic crystal [15,17-20].

\section{Pigmentary coloration}

The reflectance spectra of the yellow and red scale patches (Figure 1B,F,J,N) indicate the presence of two different, short-wavelength absorbing pigments. To identify the pigments, we immersed single scales in refractive index matching fluid $(n=1.55)$ and measured transmittance spectra with a microspectrophotometer (MSP) (Figure 3A). The differently colored scales yielded two classes of absorbance spectra (Figure 3A). The yellow, olive and green colored scales all contained a pigment with peak wavelength $\sim 390 \mathrm{~nm}$ and all red scales contained a pigment with peak wavelength $\sim 520 \mathrm{~nm}$.

We also measured with the MSP reflectance spectra of small scale areas (diameter $\sim 15 \mu \mathrm{m}$ ) of isolated single scales (Figure $3 \mathrm{~B}$ ). These spectra can be readily understood from the absorbance spectra of Figure 3A, because the pigments selectively absorb incident light in restricted wavelength ranges and thus the pigments reduce the amount of backscattered light. This is further illustrated by the scatterograms of a yellow and red scale. Illumination of a small scale area with a narrow-aperture white light beam results in both cases in a spatially wide angled, diffuse light distribution that virtually fills the whole hemisphere (Figure 3C,D).

\section{Structural coloration mechanisms}

Additionally to pigmentary coloration, three distinct forms of structural coloration occur in the wing scales of Parides butterflies: ridge multilayers (Figure $1 \mathrm{H}$ ), lumen multilayers (Figure 1L) and gyroid-type photonic crystals (Figure 1P). We have investigated the consequences of these scale structures for light reflection with scatterometry and by measuring reflectance spectra as a function of illumination angle.

\section{Ridge multilayers}

Scanning electron microscopy demonstrates that the red scales of $P$. anchises (Figure $2 \mathrm{~A}$ ) and $P$. lysander (Figure 2B) both have ridges with tilted lamellar stacks, like those of $P$. erithalion zeuxis (Figure 1G). The red scales of $P$. anchises are about flat, but those of $P$. lysander are highly curved in a gutter-like shape. The scatterograms of the two scale types, obtained with a narrow-aperture white light beam, show a wide-field distribution of red light (Figure 2C,D; see Figure 3D), but in addition they feature highly-directional blue reflections. The highly 

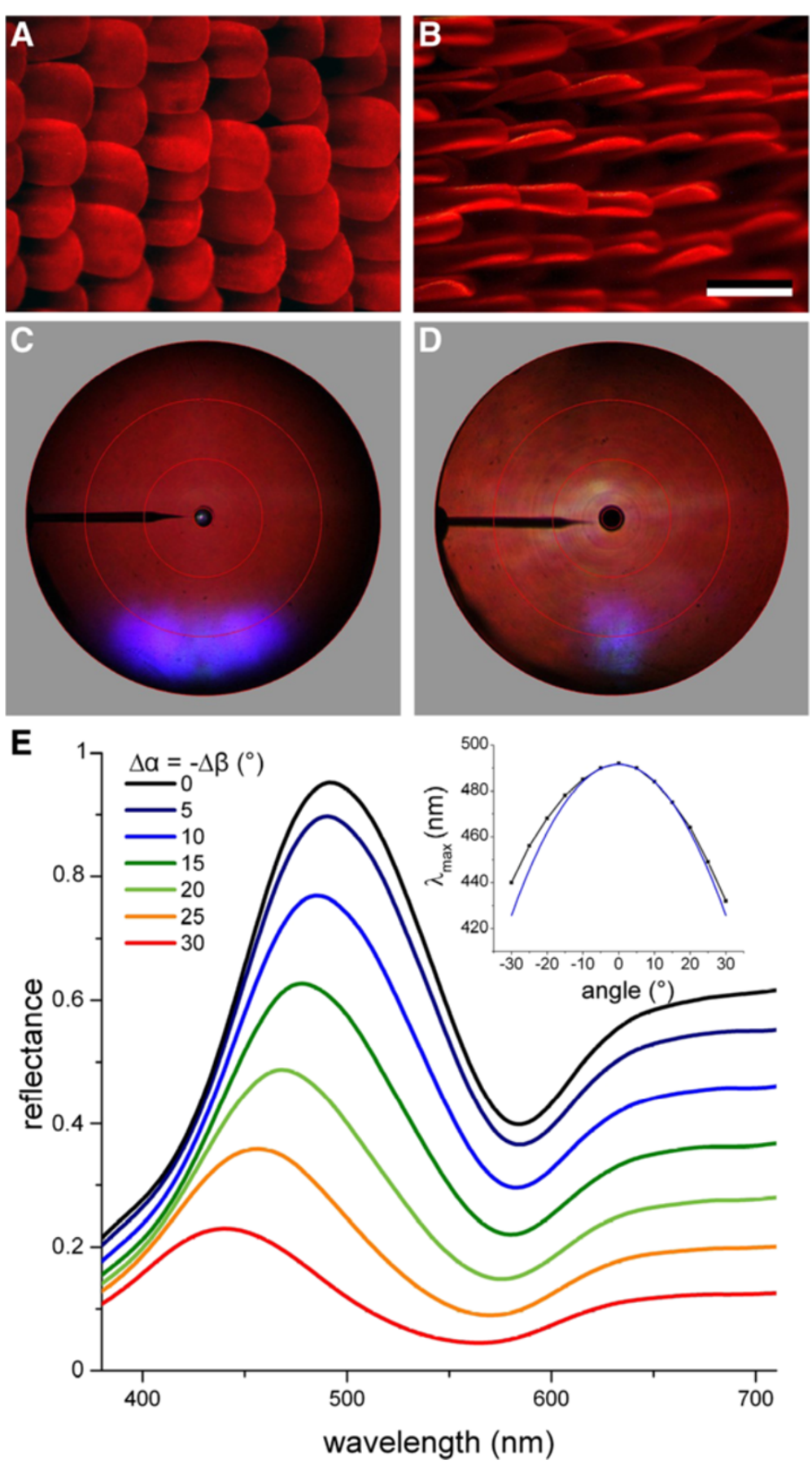

Figure 2 Red scales with blue iridescence of $P$. anchises and $P$. lysander. A, B Whereas the scales of $P$. anchises are about flat, those of $P$. lysander are highly curved. C, D Scatterograms of the two differently-shaped scales. E Angle-dependent reflectance spectra of a wing piece of $P$. anchises measured with a two-fiber goniometric setup. The illumination angle $(\Delta a)$ and detection angle $(\Delta \beta)$ were changed in steps of 5 degrees in opposite directions and symmetrically with respect to the angle of peak reflectance, which was at $\sim 60^{\circ}$ with respect to the normal to the wing plane. Inset: reflectance peak wavelength as a function of light incidence fitted with the interference condition for multilayers.

directional reflection emerges from the strongly tilted multilayers created by stacked lamellae in the scale ridges. Scale ridges with similar stacked lamellae exists in other papilionids $[25,26]$, and are even more pronounced in
Morpho [12] and pierid [27,28] butterflies. The scatterograms, which are obtained with normal illumination, show at scattering angles of $\sim 60-70^{\circ}$ that the stacks act as interference reflectors with tilt angle of $30-35^{\circ}$ with respect to 




the wing plane. The ridges are slender and they therefore diffract incident light in a plane perpendicular to the ridges (see also [29]), but the angular spread of the blue reflection of the scales of $P$. anchises (Figure 1C) is much wider than that of $P$. lysander (Figure 1D). This is obviously related to the different shape of the scales, being flat and curved, respectively.
We further investigated the angle-dependence of the blue reflection using the ARM setup, consisting of two optical fibers that can be rotated around the sample (see Methods). We first aligned the illumination and detection fiber and determined the angle where the reflectance was maximal. This was called the normal position. We then measured reflectance spectra as a function of the angle of light incidence relative to the normal position, $\Delta \alpha$, whilst the detection angle, $\Delta \beta$, was set at the mirror position: $\Delta \beta=-\Delta \alpha$ (Figure $2 \mathrm{E}$ ). When increasing the angle of incidence, the reflectance shifted hypsochromically, i.e. towards shorter wavelengths, as expected for a multilayer [30,31]. We compared the angle-dependent shift in peak wavelength derived from Figure $2 \mathrm{E}$, plotted in the inset, with values following from the interference condition for reflecting multilayers (see Methods) by using data for the thickness of the air $\left(d_{\mathrm{a}}\right)$ and cuticle layers $\left(d_{\mathrm{c}}\right)$ estimated by scanning electron microscopy: $d_{\mathrm{a}} \approx 82 \mathrm{~nm}$ and $d_{\mathrm{c}} \approx 105 \mathrm{~nm}$ (blue line). With refractive indices $n_{\mathrm{a}}=1$ and $n_{\mathrm{c}}=1.56$ a reasonable correspondence was obtained (Figure 2E, inset). The reflectance amplitude decreased with increasing angle, which is presumably due to the small size of the multilayers.

\section{Lumen multilayers}

The scales in the green forewing patches of $P$. aeneas contain a multilayer in the scale lumen, causing a brightgreen gleam (Figure $1 \mathrm{~K}, \mathrm{~L}$ ). The interference reflection by the multilayer can be eliminated by immersing the scales in refractive index fluid. Transmittance measurements on immersed green scales demonstrated the presence of the violet-absorbing pigment (Figure 3B). The scales' upper lamina is very elaborate (Figure $1 \mathrm{~L}$ ) and thus might contain a substantial fraction of the pigment (see also Additional file 1: Figure S1). Because a high pigment concentration may be expected to severely change the spatial and spectral reflectance characteristics of the wing scales [32], we investigated the reflection properties of both sides of the scales that is, of the upper, abwing side as well as the lower, adwing side, using our scatterometer and the MSP (Figure 4).

The scatterogram of the upperside shows a rather wide-field green scattering plus a clear indication of diffraction by the parallel array of ridges (Figure 4A). The scatterogram of the scale underside shows blue scattered light in a restricted spatial field (Figure 4B). The latter must represent the direct multilayer reflection. The slight spatial spread can be immediately understood from SEM observations that the scale underside is only approximately (but not perfectly) flat.

Reflectance measurements with the MSP yielded a narrow-band, green-peaking spectrum for the upperside and a much broader and more symmetrical bluegreen-peaking spectrum for the underside (Figure 4C). 

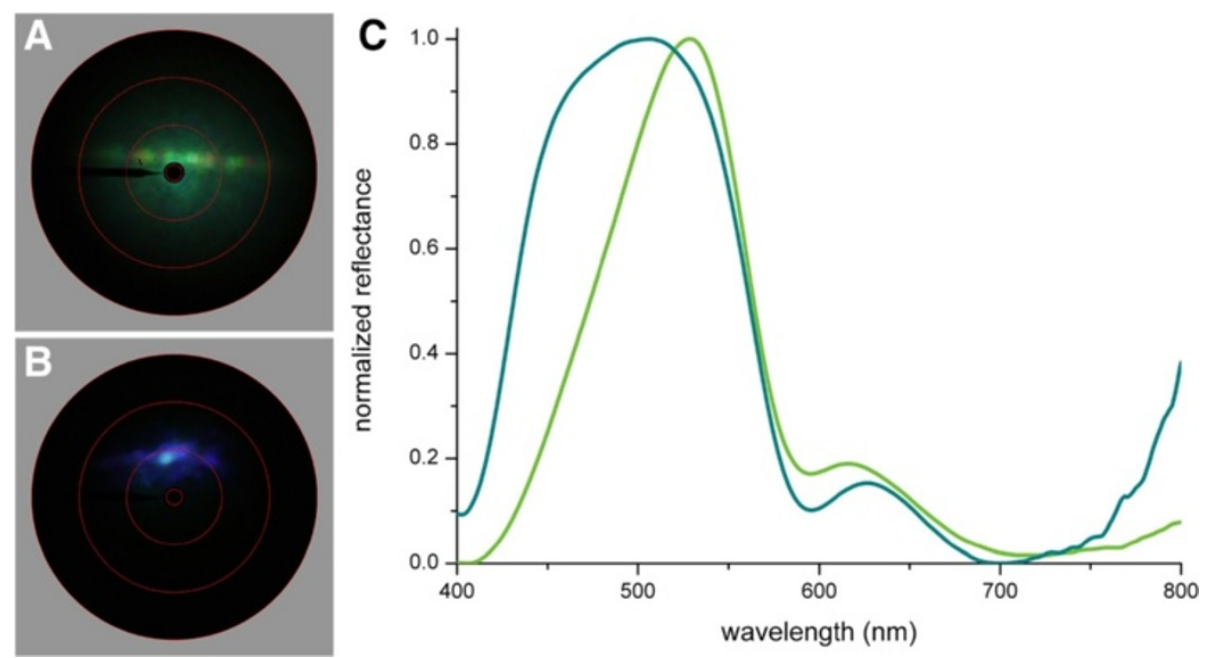

Figure 4 Scatterograms and reflectance spectra of a green cover scale of $P$. aeneas illuminated with a narrow-aperture, white-light beam. A Scatterogram of the upper side of the scale showing a diffraction pattern upon a diffuse background. B Scatterogram of the underside of the scale showing a strongly directional blue reflection. C Reflectance spectra of the upper (green curve) and under (olive curve) side of the scale.

Considering the scale's anatomy (Figure 1L), the obvious interpretation of the difference in the reflectance spectra is that the spectrum of the underside is virtually fully determined by the multilayer reflector in the scale lumen, whilst the spectrum measured from the upperside represents the multilayer's reflectance spectrum filtered by the violet-absorbing pigment.

To further investigate the angle-dependence of the reflection and scattering by the green scales of $P$. aeneas, we performed scatterometry applying wide-aperture illumination. The scatterogram of the upperside then shows a virtually uniform green color diffusely spread throughout the hemisphere above the scale (Figure 5A), while the scatterogram of the scale's underside is blue with hue depending on the spatial direction (Figure 5B). With increasing scattering angle, the color of the scattered light is blue-shifted, i.e. towards shorter wavelengths. Angle-dependent reflectance spectra from the underside measured with the scatterometer confirm the blue-shift, with a shift in peak wavelength from $\sim 485 \mathrm{~nm}$ to $430 \mathrm{~nm}$ when the angle of incident is increased from 10 to $40^{\circ}$ (Figure $5 \mathrm{C}$, red dots).

The multilayer reflectance properties can be modeled when the layer thicknesses, the refractive indices and the number of layers are known. From SEM images we estimated the layer thickness as $\sim 80 \mathrm{~nm}$ and the number of cuticle layers to be 7 . The individual layers appeared to be not entirely uniform. The chitin layers are separated by air layers, but they are connected by tiny pillars, the trabeculae, raising the effective refractive index of the air layers to $>1$. Furthermore, the chitin layers are not smooth, but locally punctured with air holes, which
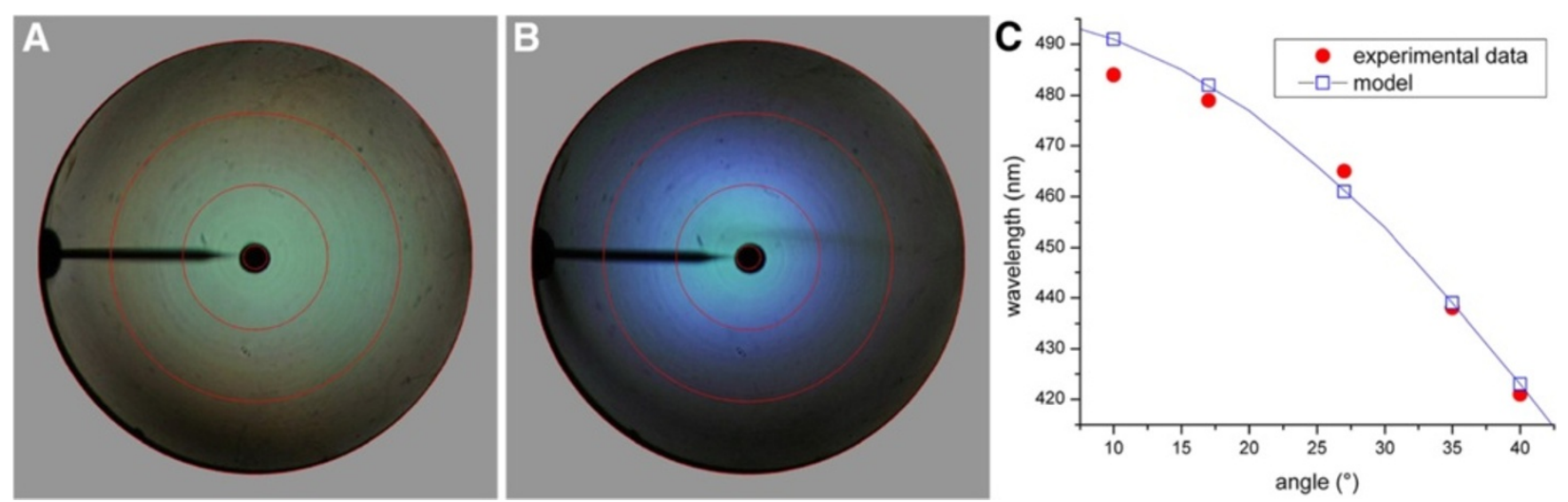

Figure 5 Scatterograms and reflectance spectra of a single green scale of Parides aeneas illuminated with a wide-aperture, white-light beam. A, B Scatterograms of the upperside and the underside of the scale with unpolarized light. C Reflectance peak wavelengths, derived from reflectance measurements, compared to peak wavelengths calculated with the interference condition of classical multilayer theory. 
reduces the effective refractive index of the cuticle layers to $<1.56$. Using refractive index values $n_{\mathrm{a}}=1.09$ and $n_{\mathrm{c}}=1.45$ (i.e. a cuticle filling fraction of 0.8 ), we could well reproduce the measured dependence of the reflectance peak wavelength on the angle of light incidence (Figure 5C and Additional file 1: Figure S2).

\section{Gyroid-type photonic crystals}

The striking green wing patches of e.g. P. childrenae and $P$. sesostris have scales with a $3 \mathrm{D}$ photonic crystal in the scale lumen with in the upper lamina a honeycombstructured layer containing the violet-absorbing pigment (Figure 1L; see also [19]). The photonic crystal layer consists of a number of differently-oriented gyroid interference reflectors, reflecting predominantly in the blue-green wavelength range [17-20]. The broader reflectance spectrum of the gyroid reflector is narrowed to the observed vivid green due to the violet-absorbing pigment in the honeycomb layer [19], in a way that is surprisingly similar to that described above for the pigmented scales with a multilayer in the lumen.

\section{Color-phylogeny correlation}

The different coloration mechanisms present in the Cattlehearts can be related to their phylogeny. The taxonomy of the family Papilionidae is generally well investigated, but only recently the genus Parides has been categorized by molecular techniques on the specieslevel with a limited number of species (18 out of 36 species). Phylogenetic information on the species-level for Parides butterflies has been published by Silva-Brandao et al. [7] based on DNA barcoding, and later slightly adjusted by Condamine et al. [4]. Further, Möhn et al. [33] classified the Cattlehearts into four major lineages depending on DNA results, habitat, external characteristics and color appearance.

We used these two classifications to map the investigated pigmentary and structural parameters onto the phylogenetic trees. Additional file 2: Table S1 lists the presence of pigments (yellow or red) and the scale structures (multilayers in the ridges and/or lumen, or gyroids) and their location on the wing (fore- and/or hindwing) for a large number of Cattlehearts. The results are summarized in Figure 6. Virtually all species possess both pigments, but a few species have only one pigment (e.g. the yellow pigment is only present in some butterflies of the chabrias group and the red pigment in some butterflies of the aeneas group of species, Figure 6, see also Additional file 2: Table S1). This strongly suggests that both pigment types are not unique for the Cattlehearts. Indeed, the wing scales of butterfly genera closely related to Parides [4], e.g. Troides and Ornithopthera, contain the same pigments, which suggests that the pigments are ancestral to all these genera.
For the structural parameters, however, the mapping shows significant differences. The classification by Condamine et al. [4], the most finely sampled phylogeny to date (Figure 6A), divides the Cattlehearts in four groups. The butterflies in groups 1 and 2 do not have a special photonic structure, but those of group 3 have either a ridge multilayer or a gyroid photonic crystal. Most of the group 4 butterflies have a multilayer in the scale lumen. The phylogeny suggests multiple independent occurrences and losses of structural characters. This is specifically obvious in the occurrence (and loss) of the ridge multilayer structures and the gyroid-type photonic crystals, which in the current phylogeny appear to have evolved and got lost multiple times within group 3 . This is a highly unlikely scenario, especially when considering the (potential) close relationship in the wing scale development of perforated multilayer structures and gyroids photonic crystals in the scale lumen (see below).

The classification by Möhn et al. [33] is rather coarsely sampled (Figure 6B). The structural parameters mostly occur in one specific group only, except for the ascanius species-group where both ridge and lumen multilayers occur. This supports a single evolutionary occurrence of these structures, much in contrast to the classification of Figure 6A. Especially in comparison with the finer sampled tree of Figure 6A, it seems likely that there has been a unique development of the trait rather than multiple independent occurrences. Thus, a species-level tree is necessary to draw specific conclusions on phylogeny.

Figure $6 \mathrm{C}$ presents an alternative phylogeny, based on the pigments and the morphological characters we identified. Albeit not being based on maximum parsimony or bootstrap methods, the tree minimizes the individual evolution of identical parameters. It will be interesting to investigate which result a combined phylogenetic analysis of morphological parameters and DNA barcoding would deliver [34].

\section{Discussion}

\section{Coloration mechanisms in the Cattlehearts}

Various coloration mechanisms are realized in the wing scales of Parides butterflies. Four different cases, each with a unique nanostructure, can be identified: i) the basic bauplan of papilionid butterflies, with a flat lower lamina and a structured upper lamina with ridges and irregular cross-ribs [23]; ii) tilted ridge lamellar stacks acting as multilayer reflectors, similar to those observed in Morpho and Pieris butterflies [12,14,28]; iii) perforated multilayer stacks in the scale lumen similar to those in lycaenid butterflies [35,36], and iv) gyroid photonic crystal layers - as also observed in lycaenids [15,18,37].

Pigments in the scales affect the scales' reflectance spectra. The ubiquitous pigment melanin, which has an absorption spectrum extending well beyond the visible 


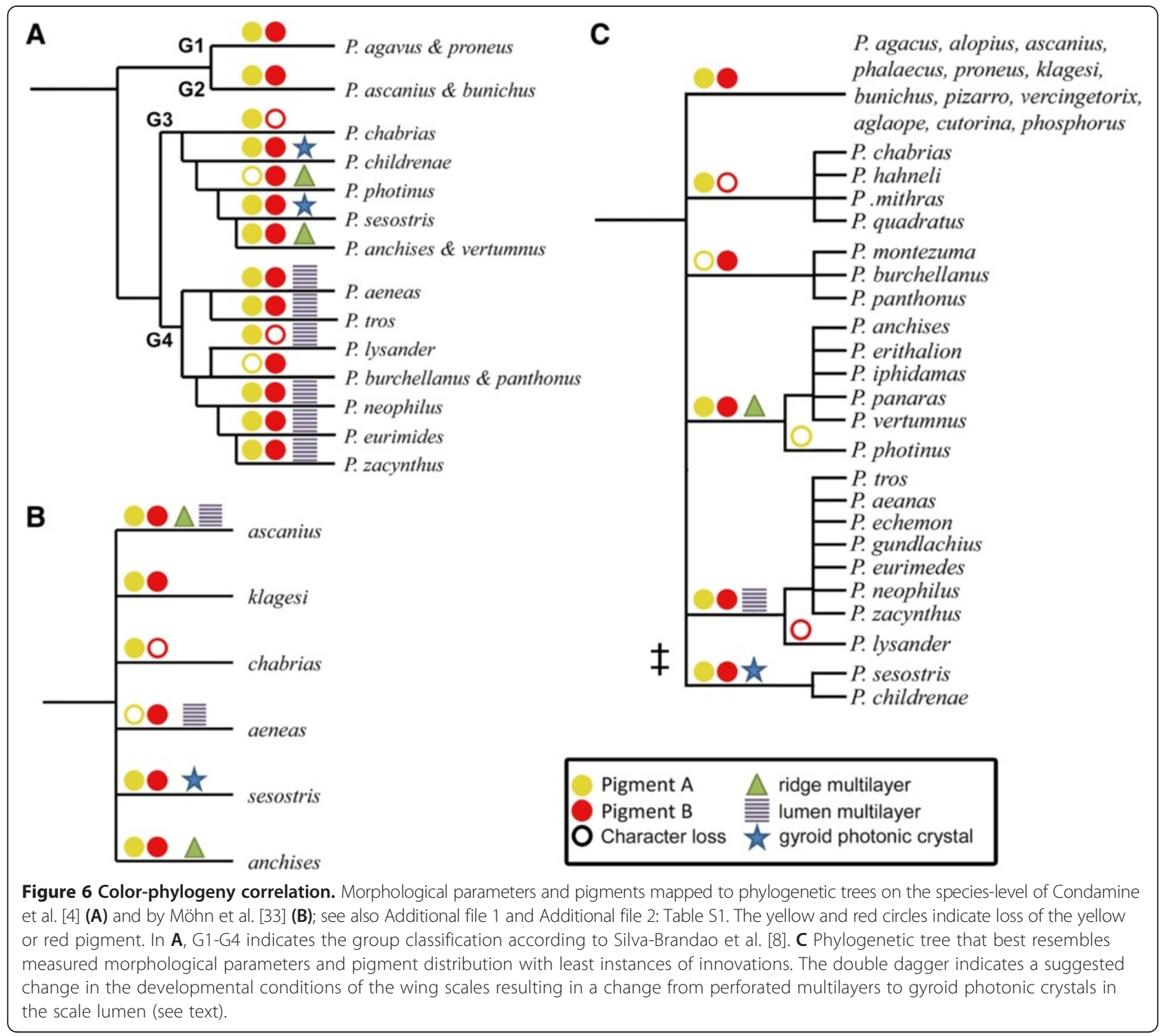

wavelength range, causes brown or black scales, depending on the pigment concentration [38]. We identified two wavelength-selective absorbing pigments embedded in the cover scales of the different Cattleheart butterflies (Figure 3). The yellow scales of P. quadratus and the green scales of several Cattlehearts contain an (ultra)violet-absorbing pigment, which is most likely the wellknown papiliochrome II of other papilionids [19,32,39]. The red scales of several Cattlehearts contain a bluegreen-absorbing pigment, which has not been specifically characterized but presumably is also a papiliochrome type pigment [40]. The pigments are located (at least partly) in the upper lamina (Additional file 1: Figure S1), which is profuse in those cases where the scale lumen contains a photonic structure, a multilayer or a 3D gyroid-type photonic crystal. Both photonic structures specifically reflect blue-green light, and in both cases the reflectance spectra are narrowed down to the green wavelength range due to spectral filtering by the pigment [19]. The upper lamina is irregular structured and thus removes the intrinsic directionality of the reflected light and makes the color mostly angle-independent (Figure 5).

We found that the scales in some Cattlehearts are distinctly curved (Figure 2). This suggests that scale curvature also tunes the directionality of the reflected light (see also [41]). Furthermore, the jet-black framing, due to heavily melanized scales, creates a contrastful wing pattern, strongly increasing the visibility of the colored wing patches, for instance for potential predators or mates.

\section{Wing scale development}

Closely related Parides species can have scales with in the lumen either perforated multilayer structures ( $P$. aeanas 
and others) or gyroid photonic crystals ( $P$. childrenae and P. sesostris, see Additional file 2: Table S1 and Figures 4, and 6). From a developmental point-of-view, it is rather difficult to assess and visualize the different stages of wing scale development. However, the self-assembly of blockcopolymers can be seen as an analogue model for the development of wing scales [18,42]. In block-copolymer systems perforated lamellar and gyroid structured phases are stable configurations lying close to each other in the phase diagram $[43,44]$. It is hence very likely that in the developing cell that develops into the butterfly wing scale, the perforated multilayers and gyroid photonic crystals are formed due to slightly different development conditions. A change in the developmental conditions, similar to e.g. a change in the concentration resulting in a phase transition in polymers [43], may thus be enough to 'switch' between the development of a perforated multilayer or a gyroid in the scale lumen.

This is the first observation of such a close connection between the lamellar phase (perforated multilayers) and the gyroid phase (photonic crystals) between two closely related species in the same genus. Additional research into the wing scale development of the Cattlehearts might therefore give important physical and chemical insight into how these complex optical structures are formed, for instance by cell-mediated self-assembly.

\section{Wing pattern and mimicry}

Many male Cattlehearts feature a similar wing patterning, and curiously, the colors of wing patches due to a multilayer or a gyroid photonic crystal in the scale lumen are nearly indistinguishable. This suggests a function of these wing patterns in mimicry rings and/or aposematic signaling, especially since many (strongly colored) Cattlehearts, like $P$. sesostris, are shunned by birds, their main predators [9].

\section{Conclusions}

We conclude that our experimental observations on the pigmentation and photonic structures in the wing scales of the Cattlehearts add important insight into the evolution of butterfly wing scales, especially given the simultaneous observation of perforated multilayers and gyroids in wing scales of butterflies within the same genus. This shows that in addition to advanced phylogenetic tools based on DNA barcoding [45], the pigmentation and morphological parameters of butterfly wing scales provide an important instrument for a detailed analysis of phylogenetic trees. Especially the highly unlikely appearance of multiple traits in closely related butterflies (Figure 6A) should be taken into account when assembling maximum likelihood phylogenetic trees and incurring on the evolution of butterflies and specifically butterfly wing traits $[34,46]$.

\section{Methods}

Animals

Specimens of the butterflies were obtained commercially trough Tropical Butterflies and Insects of America (Tampa, FL, USA) and The Bug Maniac (Makassar, Indonesia). We furthermore investigated Parides butterflies in the Lepidoptera collection of the Natural History Museum Naturalis (Leiden, the Netherlands). Additional file 2: Table S1 summarizes the investigated specimens.

\section{Photography}

The butterflies were photographed with a Canon EOS 550D camera (Canon Inc., Tokyo, Japan) equipped with a Canon $50 \mathrm{~mm}$-macro objective and a ring flash. Details of the scale arrangement on the wings were photographed with a Zeiss Universalmikroskop (Carl Zeiss AG, Oberkochen, Germany) equipped with a Kappa DX40 digital camera (Kappa GmbH, Gleichen, Germany).

\section{Spectrophotometry}

Reflectance spectra of intact wings were measured with a bifurcated probe (Avantes FCR-7UV200, Avantes, Eerbeek, The Netherlands). The probe delivered light about normally to the wing plane and the also about normally reflected light was channeled to a photodiode array spectrometer (AvaSpec-2048-2, Avantes). The light source was a Deuterium-Halogen lamp (Avantes $\mathrm{D}(\mathrm{H})-\mathrm{S}$ ). A white diffuse reference tile (Avantes WS-2) served as a reference in all reflectance measurements. Transmittance spectra obtained from isolated wing scales, immersed in a fluid with refractive index 1.55 (Cargille Labs, Cedar Grove, NJ, USA), were measured with a microspectrophotometer (MSP), being a Leitz-Ortholux microscope, equipped with the Avantes light source and spectrometer. The measured transmittance, $T(\lambda)$, yielded the absorbance (or optical density) $D(\lambda)=-\log _{10} T(\lambda)$, where $\lambda$ is the wavelength.

The angular distribution of light scattered by intact wings was measured with an angle-dependent reflectance measurement (ARM) setup, which consists of two optical fibers, mounted at two independent rotatable, but coaxial goniometers. A focused Xenon light source delivered light via one fiber to an area with diameter $\sim 4 \mathrm{~mm}$. The other fiber collected the reflected and scattered light from a wing area with diameter $\sim 9 \mathrm{~mm}$ and delivered it to the Avantes spectrometer. A UV/VIS-polarizer was mounted in front of the detection fiber (see also [31,41]). The wings were placed on a black cardboard and positioned at the collinear axes of rotation of the two goniometers. The white diffuse reference tile served as a reference.

\section{Imaging scatterometry (ISM)}

The (far-field) spatial scattering pattern of single wing scales, glued to the end of a pulled glass micropipette, was investigated with an imaging scatterometer (ISM; 
$[29,31,47])$. The ISM is built around an ellipsoidal mirror, collecting light from a full hemisphere. The investigated objects, i.e. the wing scales, positioned in the mirror's first focal point, were illuminated either with a narrow-aperture primary beam via a small hole in the ellipsoidal mirror or with a large-aperture secondary beam via a half-mirror. The far-field scattering pattern of the light backscattered by the sample was collected by a digital camera. Angle-dependent reflectance spectra were collected via a half-mirror using the Avantes spectrometer [31,47]. A small piece of magnesium oxide served as a white diffuse reference object.

\section{Scanning electron microscopy (SEM)}

The structure of the wing scales was investigated with a Philips XL30-ESEM scanning electron microscope (SEM). To prevent electric charging, the samples were sputtered with gold or palladium prior to imaging.

\section{Multilayer modeling}

The reflectance of the wing scales was modeled by applying multilayer theory [30]. The ridges responsible for the scattering of light consist of alternating air (a) and cuticle (c) layers, with thickness $d_{\mathrm{a}}$ and $d_{\mathrm{c}}$ and refractive index $n_{\mathrm{a}}$ and $n_{\mathrm{c}}$. Although the cuticle refractive index is slightly dispersive [48], we have assumed for all wavelengths $n_{\mathrm{a}}=1.0$ and $n_{c}=1.56$, to simplify the analysis. The propagation of a light beam through the multilayer can be found from Snell's law: $n_{\mathrm{a}} \sin \theta_{\mathrm{a}}=n_{\mathrm{c}} \sin \theta_{\mathrm{c}}$, where $\theta_{\mathrm{a}}$ and $\theta_{\mathrm{c}}$ are the angles of incidence in air and cuticular medium. The wavelength of peak reflectance of a multilayer is given by the interference condition $\lambda_{\max }=2\left(n_{\mathrm{a}} d_{\mathrm{a}} \cos \theta_{a}+n_{\mathrm{c}} d_{\mathrm{c}} \cos \theta_{\mathrm{c}}\right)[49,50]$.

\section{Taxonomy}

We have used the species-level taxonomy provided by Condamine et al. ([4]; slightly updated from [7]), where 17 species have been examined on the species-level by DNA barcoding, and the taxonomy provided by Möhn et al. [33], where 36 species have been grouped (for more details, see Additional file 1).

\section{Data accessibility}

The data set supporting the results of this article is included within the article and its additional files.

\section{Additional files}

Additional file 1: Figures S1 and S2 and additional details on the taxonomy.

Additional file 2: Table S1. Investigated Cattlehearts, morphological parameters and pigments found in the wing scales.

\section{Competing interests}

The authors declare that they have no competing interests.

\section{Authors' contributions}

BDW conceived the design of the study, BDW and NY carried out the experiments, DGS participated in the design and coordination of the study, BDW and DGS drafted the manuscript. All authors read and approved the final manuscript.

\section{Acknowledgements}

We thank Zsolt Bálint, Casper van der Kooi and two anonymous reviewers for valuable comments on the manuscript and Hein Leertouwer for collaboration. This study was financially supported by the Air Force Office of Scientific Research/European Office of Aerospace Research and Development (Grant FA8655-08-1-3012 to D.G.S.).

\section{Author details}

${ }^{1}$ Computational Physics, Zernike Institute for Advanced Materials, University of Groningen, Nijenborgh 4, NL-9747AG Groningen, The Netherlands. ${ }^{2}$ Present address: Department of Physics, Cavendish Laboratories, University of Cambridge, $13 \mathrm{JJ}$ Thomson Avenue, CB3 OHE Cambridge, UK. ${ }^{3}$ Present address: Accenture Nederland B.V, Gustav Mahlerplein 90, NL-1082 MA Amsterdam, The Netherlands.

Received: 2 April 2014 Accepted: 14 July 2014 Published: 27 July 2014

\section{References}

1. Doucet SM, Meadows MG: Iridescence: a functional perspective. $J R$ SoC Interface 2009, 6(Suppl 2):S115-S132.

2. Stevens M, Merilaita S: Animal camouflage: mechanisms and function. Cambridge, UK: Cambridge University Press; 2011.

3. Andersson M: Sexual selection. Princeton, NJ: Princeton University Press; 1994.

4. Condamine FL, Sperling FAH, Wahlberg N, Rasplus J, Kergoat GJ: What causes latitudinal gradients in species diversity? Evolutionary processes and ecological constraints on swallowtail biodiversity. Ecol Lett 2012, 15:267-277.

5. Ehrlich PR, Raven PH: Butterflies and plants: a study in coevolution. Evolution 1964, 18:586-608.

6. Simonsen TJ, Zakharov EV, Djernaes M, Cotton AM, Vane-Wright RI, Sperling $\mathrm{FAH}$ : Phylogenetics and divergence times of Papilioninae (Lepidoptera) with special reference to the enigmatic genera Teinopalpus and Meandrusa. Cladistics 2011, 27:113-137.

7. Silva-Brandao KL, Freitas AV, Brower AV, Solferini VN: Phylogenetic relationships of the New World Troidini swallowtails (Lepidoptera: Papilionidae) based on COI, COIl, and EF-1alpha genes. Mol Phylogen Evol 2005, 36:468-483.

8. Silva-Brandao KL, Azeredo-Espin AM, Freitas AV: New evidence on the systematic and phylogenetic position of Parides burchellanus (Lepidoptera: Papilionidae). Mol Ecol Res 2008, 8:502-511.

9. Pinheiro CEG: Palatability and escaping ability in Neotropical butterflies: tests with wild kingbirds (Tyrannus melancholicus, Tyrannidae). Biol J Linn Soc 1996, 59:351-365.

10. Ruxton GD, Sherratt TN, Speed MP: Avoiding Attack: The Evolutionary Ecology of Crypsis, Warning Signals and Mimicry. Oxford University Press; 2004. ISBN 0-19-852859-0.

11. Pinheiro CEG: Asynchrony in daily activity patterns of butterfly models and mimics. J Trop Ecol 2007, 23:119-123.

12. Kinoshita S: Structural colors in the realm of nature. Singapore: World Scientific; 2008.

13. Ghiradella $\mathrm{H}$ : Light and color on the wing: structural colors in butterflies and moths. Appl Opt 1991, 30:3492-3500.

14. Vukusic P, Sambles JR: Photonic structures in biology. Nature 2003, 424:852-855.

15. Michielsen K, Stavenga DG: Gyroid cuticular structures in butterfly wing scales: biological photonic crystals. J R Soc Interface 2008, 5:85-94.

16. Vukusic P: Advanced photonic systems on the wing-scales of Lepidoptera. In Functional surfaces in biology - Little structures with big effects, Volume 1. Edited by Gorb SN. New York: Springer; 2009:237-258.

17. Poladian L, Wickham S, Lee K, Large MC: Iridescence from photonic crystals and its suppression in butterfly scales. J R Soc Interface 2009, 6(Suppl 2):S233-S242. 
18. Saranathan V, Osuji CO, Mochrie SG, Noh H, Narayanan S, Sandy A, Dufresne ER, Prum RO: Structure, function, and self-assembly of single network gyroid $(14,32)$ photonic crystals in butterfly wing scales. Proc Natl Acad Sci U S A 2010, 107:11676-11681.

19. Wilts BD, Michielsen K, De Raedt H, Stavenga DG: Iridescence and spectral filtering of the gyroid-type photonic crystals in Parides sesostris wing scales. Interface Focus 2012, 2:681-687.

20. Yoshioka S, Fujita H, Kinoshita S, Matsuhana B: Alignment of crystal orientations of the multi-domain photonic crystals in Parides sesostris wing scales. J R Soc Interface 2014, 11:20131029.

21. Vukusic P, Stavenga DG: Physical methods for investigating structural colours in biological systems. J R Soc Interface 2009, 6(Suppl 2):S133-S148.

22. Vukusic $P$, Sambles JR, Lawrence CR: Structurally assisted blackness in butterfly scales. Proc R Soc B 2004, 271(Suppl 4):S237-S239.

23. Ghiradella H: Insect cuticular surface modifications: scales and other structural formations. Adv Insect Physiol 2010, 38:135-180.

24. Ghiradella $\mathrm{H}$ : Structure and development of iridescent butterfly scales: lattices and laminae. J Morphol 1989, 202:69-88.

25. Dechkrong $P$, Jiwajinda S, Dokchan P, Kongtungmon M, Chomsaeng N, Chairuangsri T, Yu C, Hsiao C, Shiojiri M: Fine structure of wing scales of butterflies, Euploea mulciber and Troides aeacus. J Struct Biol 2011, 176:75-82.

26. Vukusic P, Sambles JR, Lawrence CR, Wootton RJ: Structural colour: now you see it-now you don't. Nature 2001, 4:36.

27. Giraldo MA, Yoshioka S, Stavenga DG: Far field scattering pattern of differently structured butterfly scales. J Comp Physiol A 2008, 194:201-207.

28. Wilts BD, Pirih P. Stavenga DG: Spectral reflectance properties of iridescent pierid butterfly wings. J Comp Physiol A 2011, 197:693-702.

29. Stavenga DG, Leertouwer HL, Pirih P, Wehling MF: Imaging scatterometry of butterfly wing scales. Opt Express 2009, 17:193-202.

30. Yeh P: Optical waves in layered media. Hoboken NJ: Wiley-Interscience; 2005.

31. Stavenga DG, Wilts BD, Leertouwer $\mathrm{HL}$, Hariyama T: Polarized iridescence of the multilayered elytra of the Japanese jewel beetle, Chrysochroa fulgidissima. Phil Trans Roy Soc B 2011, 366:709-723.

32. Wilts BD, Trzeciak TM, Vukusic P, Stavenga DG: Papiliochrome II pigment reduces the angle-dependency of structural wing colouration in nireus group papilionids. J Exp Biol 2012, 215:796-805.

33. Möhn E, Bauer E, Frankenbach T: Butterflies of the world: Papilionidae XIII: Parides. Canterbury: Goecke \& Evers; 2007.

34. Simonsen TJ, de Jong R, Heikkilä M, Kaila L: Butterfly morphology in a molecular age - does it still matter in butterfly systematics? Arthropod Struct Dev 2012, 41:307-322.

35. Wilts BD, Leertouwer HL, Stavenga DG: Imaging scatterometry and microspectrophotometry of lycaenid butterfly wing scales with perforated multilayers. J R Soc Interface 2009, 6(Suppl 2):S185-S192

36. Bálint Z, Kertész K, Piszter G, Vértesy Z, Biró LP: The well-tuned blues: the role of structural colours as optical signals in the species recognition of a local butterfly fauna (Lepidoptera: Lycaenidae: Polyommatinae). J R Soc Interface 2012, 9:1745-1756.

37. Michielsen K, De Raedt H, Stavenga DG: Reflectivity of the gyroid biophotonic crystals in the ventral wing scales of the Green Hairstreak butterfly, Callophrys rubi. J R Soc Interface 2010, 7:765-771.

38. Stavenga DG, Leertouwer HL, Hariyama T, De Raedt H, Wilts BD: Sexual dichromatism of the damselfly Calopteryx japonica caused by a melanin-chitin multilayer in the male wing veins. PLoS One 2012, 7:e49743.

39. Umebachi Y: Yellow pigments in the wings of Papilio xuthus (papilionid butterfly). Acta Vitaminologica et Enzymologica 1975, 29:219-222.

40. Umebachi Y: Papiliochrome, a new pigment group of butterfly. Zool Sci 1985, 2:163-174

41. Pirih P, Wilts BD, Stavenga DG: Spatial reflection patterns of iridescent wings of male pierid butterflies: curved scales reflect at a wider angle than flat scales. J Comp Phys A 2011, 197:987-997.

42. Ghiradella H: Structure and development of iridescent lepidopteran scales - the Papilionidae as a showcase family. Ann Entomol Soc 1985, 78:252-264.

43. Hamley IW, Castelletto V, Mykhaylyk OO, Yang Z, May RP, Lyakhova KS, Sevink GJ, Zvelindovsky AV: Mechanism of the transition between lamellar and gyroid phases formed by a diblock copolymer in aqueous solution. Langmuir 2004, 20:10785-10790.
44. Cochran EW, Garcia-Cervera CJ, Fredrickson GH: Stability of the gyroid phase in diblock copolymers at strong segregation. Macromolecules 2006, 39:2449-2451

45. Reed RD, Papa R, Martin A, Hines HM, Counterman BA, Pardo-Diaz C, Jiggins CD, Chamberlain NL, Kronforst MR, Chen R, Halder G, Nijhout HF, McMillan WO: Optix drives the repeated convergent evolution of butterfly wing pattern mimicry. Science 2011, 333:1137-1141.

46. Beutel RG, Kristensen NP: Morphology and insect systematics in the era of phylogenomics. Arth Struct Devel 2012, 41:303-305.

47. Wilts BD, Michielsen K, De Raedt H, Stavenga DG: Hemispherical Brillouin zone imaging of a diamond-type biological photonic crystal. J R Soc Interface 2012, 9:1609-1614.

48. Leertouwer HL, Wilts BD, Stavenga DG: Refractive index and dispersion of butterfly chitin and bird keratin measured by polarizing interference microscopy. Opt Express 2011, 19:24061-24066.

49. Land MF: The physics and biology of animal reflectors. Prog Biophys Mol Biol 1972, 24:77-105.

50. Kinoshita S, Yoshioka S, Miyazaki J: Physics of structural colors. Rep Prog Phys 2008, 71:076401.

doi:10.1186/s12862-014-0160-9

Cite this article as: Wilts et al:: Pigmentary and photonic coloration mechanisms reveal taxonomic relationships of the Cattlehearts (Lepidoptera: Papilionidae: Parides). BMC Evolutionary Biology 2014 14:160.

\section{Submit your next manuscript to BioMed Central and take full advantage of:}

- Convenient online submission

- Thorough peer review

- No space constraints or color figure charges

- Immediate publication on acceptance

- Inclusion in PubMed, CAS, Scopus and Google Scholar

- Research which is freely available for redistribution 